\title{
Degassing/oxidation process at Aso volcano inferred by the color of ash
}

\author{
ISOJI MIYAGI ${ }^{1}$, NOBUO GESHI $^{1}$, HIDEO HOSHIZUMI ${ }^{1}$
}

${ }^{1}$ Geological Survey of Japan, 1-1-1-7, Higashi, Tsukuba, Ibaraki, 305-8567, JAPAN

The oxidation state of iron in volcanic ash has the potential as a tool to detect degassing and oxidation processes of magma; the iron oxidizes either by atmospheric oxygen or by dehydrogenation degassing of dissolved water. We focused on color, delegate of the oxidation state, of volcanic ash to understand the temporal change of the magma oxidation and degassing during the activity of Aso Nakadake No.1 crater from November 2014 to May 2015. We measured the color of fine-sized (silt or less) components of volcanic ash, separated by elutriation, using a soil color meter. Also, sieved coarse-sized components (particle size 0.25-0.5 mm), ground for about 30 minutes in an agate mortar, are measured in some cases. The 'a*' value (intensity of redness, CIE 1976) of fine-sized components of the volcanic ash showed a systematic change over time. Specifically, the value increased significantly from November 25,2014 , to December $4(0.3 \rightarrow$ 6.7). It decreased significantly to December $24(6.7 \rightarrow 1.7)$. In contrast, the value of the coarse-sized component of volcanic ash decreased from 0.94 (November 29, 2014) to 0.44 (December 3). The color of the fine particles of volcanic ash to reflect the temperature of the plume, while the color of the coarse particles is likely to reflect the oxidation state of magma in the conduit before eruption (Miyagi et al., 2010, BVSJ). While the oxidation occurs preferentially for finer particles with a large specific surface area, oxidation due to dehydrogenation can also occur before magma fragmentation in a conduit without interaction with air. From late November to early December 2014, the fine-grained component of volcanic ash changed to an oxidative color. Conversely, the coarse component changed to a reductive color tone, suggesting an increase in plume temperatures and an increase in reducible underground magma supply, which is supported by the component analysis of the volcanic ash. The ' ${ }^{*}$ ' value of the fine-grained component remains low, in spite of the higher temperature expected, suggesting that there is some mechanism that inhibits the oxidation of volcanic ash particles in the plume. We propose a model in which a large proportion of volcanic gas to ash emitted from the crater lowers the amount of air mixed to obtain buoyancy of volcanic cloud, and as a result, oxidation of volcanic ash by atmospheric oxygen may not have progressed. 